\title{
Identification of copper ions in aqueous and vitreous of eyes containing copper and iron foreign bodies*
}

\author{
Y. YASSUR AND H. ZAUBERMAN \\ From the Department of Ophthalmology, Hadassah University Hospital, ferusalem \\ AND M. ZIDON \\ From the Department of Inorganic Chemistry, The Hebrew University, ferusalem
}

Intraocular foreign bodies have nowadays become a frequent problem in war and in some industrial countries. The extraction of magnetic foreign bodies does not usually pose a serious problem. However, removal of nonmagnetic metallic foreign bodies involves a serious risk to the eye and their extraction is justified only when the metal is chemically toxic to the eye, such as copper. The proper identification of an intraocular metallic foreign body is therefore important.

Several methods of identifying metallic intraocular foreign bodies have been tried: polarography, (Krwawicz, Zagorski, and Szari, I966); colorimetric chemical reaction (Gerhard, I966); neutron activation of copper (Bottger, Fittkan, Niese, and Hanson, 1968); and atomic absorption spectrophotometry (Runyan and Levri, 1970). The lastnamed authors described the analysis of vitreous samples from rabbits and humans to determine quantitatively copper and iron ions using the Perkin-Elmer 303 atomic absorption spectrophotometer. However, the aspiration of vitreous from affected human eyes is sometimes a dangerous procedure.

The present study was undertaken to estimate the reliability of aqueous analysis, and as copper is one of the most dangerous nonmagnetic metals, it was used as a model in the experiments. The study had three purposes:

I. To examine if copper ions could be discovered in the aqueous of eyes containing copper particles in the vitreous.

2. To investigate the relative quantities of copper ions in the aqueous and vitreous of such eyes.

3. To determine whether aqueous copper ions increase only in eyes containing copper particles or if this also occurs in eyes containing iron particles.

*'This work was supported by a grant from Ophthalmology Research Fund, Switzerland, and by the Rosenthal-Statler Foundation

Address for reprints: Y. Yassur, MD, Department of Ophthalmology, Beilinson Hospital, Petach Tikvah, Israel

\section{Material and methods}

Altogether 33 albino rabbits weighing 3-4 $\mathrm{kg}$ were used in the study. They were divided into three groups. The first group comprised 17 rabbits, and the second and third eight rabbits each.

Each animal was anaesthetized with an intravenous injection of pentobarbital $0.25 \mathrm{mg} / \mathrm{kg}$. The pupils were dilated with scopolamine 0.25 per cent and neosynephrine to per cent, and the eyes were proptosed.

In the first and second groups pure sterile copper particles were introduced. Each particle weighed 8-II $\mathrm{mg}$, its surface area being about $10 \mathrm{~mm}^{2}$. A single particle was injected into the vitreous of each eye through the pars plana with a no. 20 lumbar puncture needle. Atropine and antibiotic ointment were applied to the eyes after the injection.

In the third group iron particles of similar sizes and weights were injected using a similar technique.

In the first group, the relationship between the copper ion content of the aqueous and vitreous was determined at different intervals. For this purpose six eyes were enucleated after $1,3,5,8$, and 12 days. In each enucleated eye, $0.2 \mathrm{ml}$ aqueous was removed by a no. 25 needle attached to a metal-free syringe and put into a clean metal-free test tube. The eye was then opened and $0.6 \mathrm{ml}$ vitreous was withdrawn into a similar test tube. The vitreous cavity was examined for signs of infection and the degree and extent of encapsulation of the foreign body were measured. The aqueous and vitreous samples were analysed separately for copper ion content by the Perkin-Elmer 303 atomic absorption spectrophotometer with the hollow cathode lamp.

In the second group, the quantity of copper in the aqueous was determined in vivo at different times during the 4 weeks after implantation. Every animal was anaesthetized 2 and 4 days, and 1,2 , and 4 weeks after surgery, and samples of $0.2 \mathrm{ml}$ aqueous were taken each time for copper ion content analysis using the same spectrophotometric technique.

In the third group, the copper ion content in the aqueous and vitreous of eyes containing iron particles was determined. Six eyes were enucleated 4 and 7 days after surgery for implantation. Aqueous and vitreous samples were obtained as for the first group.

The baseline for copper ion content in aqueous and vitreous was determined for each group by two rabbits 
Table I Copper ion content of vitreous and aqueous of eyes containing 8 to I I mg intravitreal pure copper particles

\begin{tabular}{|c|c|c|c|c|c|c|}
\hline \multirow[t]{2}{*}{ Eye } & \multicolumn{5}{|l|}{ Days } & \multirow[t]{2}{*}{ Baseline } \\
\hline & I & 3 & 5 & 8 & 12 & \\
\hline $\begin{array}{l}\text { Vitreous } \\
\text { Aqueous }\end{array}$ & $\begin{array}{l}0.90 \pm 0.08 \\
0.15 \pm 0.01\end{array}$ & $\begin{array}{l}1 \cdot 50 \pm 1 \cdot 03 \\
0.65 \pm 0.05\end{array}$ & $\begin{array}{l}1 \cdot 70 \pm 1 \cdot 05 \\
0.90 \pm 0.70\end{array}$ & $\begin{array}{l}3 \cdot 10 \pm 1 \cdot 05 \\
1 \cdot 40 \pm 1 \cdot 01\end{array}$ & $\begin{array}{l}7 \cdot 40 \pm 5 \cdot 08 \\
1 \cdot 55 \pm 1 \cdot 04\end{array}$ & $\begin{array}{l}0.20 \pm 0.08 \\
0.10 \pm 0.05\end{array}$ \\
\hline
\end{tabular}

Mean values and standard deviations are given in parts/million

Each column represents results from six different eyes

Baseline represents values of copper in four eyes in which surgery was performed without foreign body implantation

that underwent the surgical procedure of puncturing the pars plana but without introducing a foreign body into the vitreous.

\section{Results}

The results for the first group of animals are summarized in Table I and Fig. I. Samples of vitreous and aqueous obtained I day after the foreign body implantation showed no significant increase in copper ions when compared with the baseline values obtained from the controls. Between the third day and the end of the second week after implantation copper values rose steadily, those in the vitreous being two to four times higher than those in the aqueous. Fig. I shows that by the eighth day after implantation the rate of rise in the vitreous was much higher than that in the aqueous.

The results for the second group are given in Table II and Fig. 2. Between the second day and the end of the fourth week there was a rise in the content of copper ions in the aqueous. A moderate decline in the mean value had occurred by the end of the second week and the copper values rose during the third and fourth week.

The results for the third group are given in Table III and Fig. 3. In eyes containing an iron foreign body the copper ion content in the vitreous and the aqueous also rose during the first week after implantation compared with the baseline values.

Standard deviations were high for most of the groups.

\section{Discussion}

When an eye harbours a copper foreign body in the vitreous, an increase in the copper ions occurs not only in the vitreous but also in the aqueous of that eye. Aspiration of aqueous from eyes in which the nature of the intraocular foreign body is unknown is relatively a simpler and safer procedure than aspiration of vitreous. For the atomic absorption spectrophotometry technique $0.2 \mathrm{ml}$ aqueous are

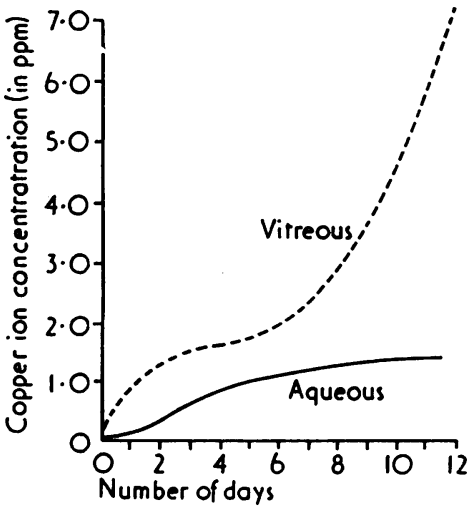

(I)

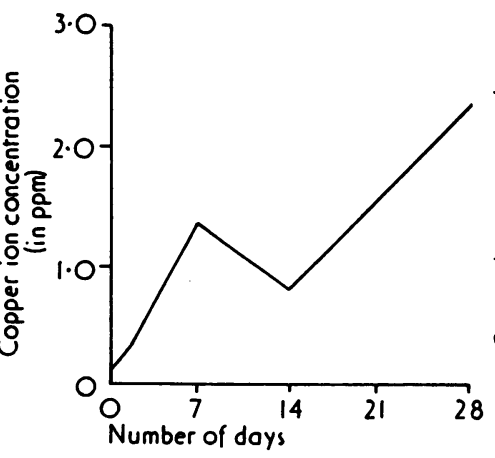

(2)

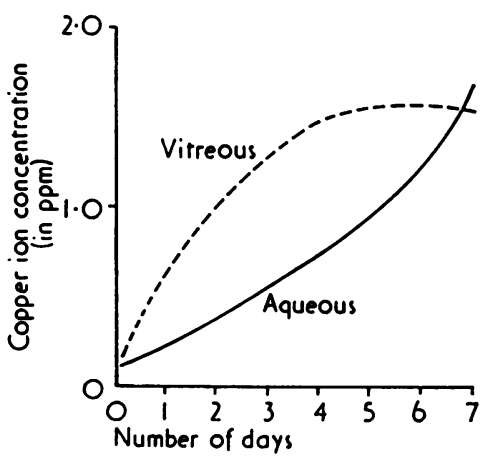

(3)

FIG. I Relative rise of copper ion concentration in aqueous and vitreous of albino rabbits during first 2 weeks after intravitreal implantation of pure copper particles

FIG. 2 Rise in copper ion concentration in aqueous of albino rabbits during first month after intravitreal implantation of pure copper foreign body

FIG. 3 Rise of copper ions in aqueous and vitreous of albino rabbits during first week after intravitreal implantation of pure iron foreign bodies 
Table II Analysis of aqueous copper in 12 eyes containing 8 to I I $m g$ intravitreal pure copper particles

\begin{tabular}{|c|c|c|c|c|c|}
\hline \multicolumn{2}{|l|}{ Days } & \multicolumn{3}{|l|}{ Weeks } & \multirow[t]{2}{*}{ Baseline } \\
\hline 2 & 4 & $\mathbf{I}$ & 2 & 4 & \\
\hline $0.31 \pm 0.18$ & $0.72 \pm 0.31$ & $1 \cdot 33 \pm 0 \cdot 40$ & $0.81 \pm 0.64$ & $2 \cdot 36 \pm 0.46$ & $0.14 \pm 0.01$ \\
\hline
\end{tabular}

Samples were taken in vivo successively from all 12 eyes at intervals of 2 days to 4 weeks

Mean values and standard deviations are given in parts/million

Baseline represents values of aqueous copper in four eyes in which surgery was performed without foreign body implantation

Table III Copper ion content of vitreous and aqueous of 12 eyes containing 8 to I I mg intravitreal pure iron particles 4 and 7 days after implantation of foreign body

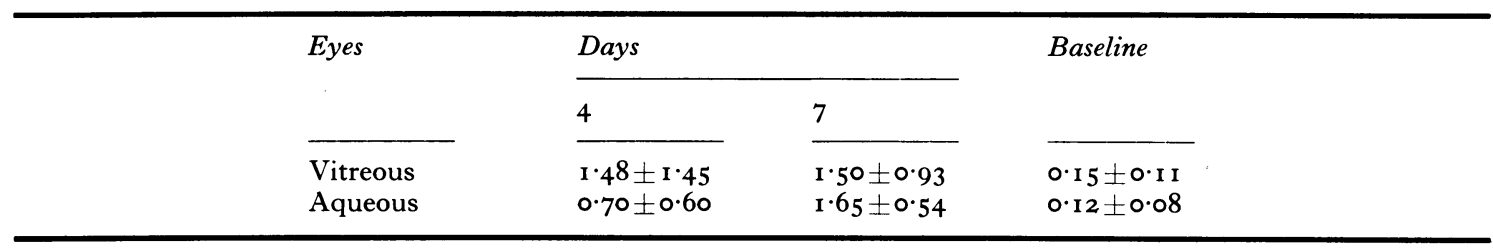

Each column represents results obtained from six different eyes

Mean values and standard deviations are given in parts/million

Baseline represents values of copper in four eyes in which surgical procedure was performed without foreign body implantation

sufficient to detect minute quantities of copper ions measured in parts per million, and these are of diagnostic value. The concentration of ions in the aqueous was found to be lower than in the vitreous although it was still well above the baseline in most of the experimental eyes. The difference in concentration of copper ions between aqueous and vitreous may depend on such factors as the direct corrosive effect of the vitreous on the metal lodged in it, and a more rapid turnover of fluid in the anterior chamber.

In certain eyes the values of copper ions did not increase in vitreous or aqueous, nor did they increase after a preliminary rise. This can be related to encapsulation of the foreign body, and the clinical significance of such a finding can be of importance since it may indicate less toxicity or none.

In judging the copper ion content of an eye containing unidentified metal it has to be romembered that eyes containing iron particles in the vitreous also show a rise of copper ion content in both aqueous and vitreous. This may be explained by some biological mechanism which alters the copper ion metabolism, like altering the bloodvitreous or blood-aqueous barrier, but the nature of this phenomenon is not clear. This rise did not occur with eyes which underwent the surgical procedure only (baseline eyes).

Clinically, this important source of error can be checked by positive magnetic response of the unidentified intraocular foreign body.

These false negative and false positive possibilities should be borne in mind when trying to judge the nature of an intraocular foreign body in an eye in which the aqueous shows a rise in copper ion content as measured by the atomic absorption spectrophotometer.

\section{Summary}

The reliability of aqueous analysis by the atomic absorption spectrophotometer for copper ion content in samples from eyes containing intravitreal pure copper particles was investigated. It was demonstrated that values of copper ions rose well above the normal in the aqueous as well as in the vitreous of such eyes. The standard deviations of the mean values were generally high. It was also found that a similar copper ion content increase occurred in eyes containing iron particles. The possible clinical implications of these findings are discussed.

\section{References}

BOtTGer, M., fitTKan, S., NIESE, S., and hanson, h. (I968) Acta biol. med. germ., 21, IO I

GERHARD, I. P. (I966) Docum. ophthal. (Den Haag), 20, ro4

KRWAWICZ, T., ZAgORSKI, K., and SZARI, B. (1966) Pol. med. F., 5, 466

RUNYAN, T. H., and LEVRI, E. A. (1970) Amer. F. Ophthal., 69, r053 